Published in final edited form as:

Dig Liver Dis. 2009 September ; 41(9): 676-682. doi:10.1016/j.dld.2009.01.002.

\title{
Practice patterns in screening for varices: An American survey
}

\author{
A.S. Barritt IVa, ${ }^{*}$ and M.R. Arguedas ${ }^{b,{ }^{*}}$ \\ a Division of Gastroenterology and Hepatology, University of North Carolina-Chapel Hill, United \\ States \\ ${ }^{b}$ Department of Medicine, Baptist Medical Center South, University of Alabama at Birmingham- \\ Montgomery Campus, United States
}

\begin{abstract}
Background and aims-Guidelines recommend screening for gastroesophageal varices. Regional studies suggest screening is underutilized, but information from across the United States is unavailable. We explored practice patterns and adherence to guidelines in a random sample of physicians and sought to define whether differences existed according to practice type, setting and years of practice.
\end{abstract}

Materials and Methods-Surveys were randomly sent to 600 gastroenterologists and hepatologists. Descriptive data is presented as percentage and comparisons were performed by chi-square analysis. Significance was defined at a $p$ value $<0.05$.

Results -180 completed surveys were returned. Mean age was $48.9 \pm 10$ years and $87 \%$ were male. $50 \%$ were community-based and $74 \%$ had been in practice $>10$ years. $53 \%$ (78\% hepatologists versus $45 \%$ of gastroenterologists) screened consistently ( $>75 \%$ of the time), $(p<$ 0.001 ). No differences in screening frequency were found according to practice setting or years in practice. $62 \%$ screened all cirrhotics whereas $38 \%$ screened based on clinical characteristics. In patients without gastroesophageal varices, $60 \%$ repeated esophagogastroduodenoscopy in 2-3 years. In those with small gastroesophageal varices, repeat esophagogastroduodenoscopy was recommended in 1-2 years by $73 \%$. In patients with small and large varices, $40 \%$ and $54 \%$ of physicians respectively, recommended prophylaxis. $6 \%$ of physicians recommend prophylaxis regardless of the presence or size of varices.

Conclusions-Screening for varices is under-implemented. Many screened based on clinical findings that have not been shown to reliably predict high-risk gastroesophageal varices. Continued education and removal of financial barriers to screening are central to increasing screening rates and improving patient outcomes.

\section{Keywords}

Cirrhosis; Guidelines; Prevention; Primary prophylaxis; Surveillance

\footnotetext{
${ }^{\star}$ This research was performed while Dr. Barritt was a member of the Department of Medicine and Dr. Arguedas was a member of the Liver Center at The University of Alabama-Birmingham.

(C) 2009 Editrice Gastroenterologica Italiana S.r.l. Published by Elsevier Ltd. All rights reserved.

"Corresponding author at: UNC Gastroenterology and Hepatology, Campus Box 7080, Chapel Hill, NC 27599-7080, United States. Tel.: +1 919843 1002; fax: +1 919843 3521. abarritt@unch.unc.edu (A.S. Barritt IV).

Conflict of interest statement

None declared.
} 


\section{Introduction}

Haemorrhage from gastroesophageal varices (GEV) is the most lethal complication of cirrhosis and portal hypertension. Variceal bleeding (VB) occurs in $25-40 \%$ of patients with cirrhosis and each episode of bleeding is associated with a $10-30 \%$ mortality rate $[1,2]$ In light of the frequency with which VB occurs in patients with cirrhosis and the high rate of mortality associated with each bleeding episode, prevention of bleeding is an important goal in the management of patients with cirrhosis.

Primary prophylaxis, through both pharmacological and endoscopic means, reduces the incidence of variceal haemorrhage in patients with cirrhosis. Multiple studies have shown that beta-blockers reduce the risk of bleeding by nearly $50 \%[3,4]$ While there are recent data that beta-blockers reduce bleeding when given to patients with small varices [5], most studies only demonstrate a benefit of primary prophylaxis for patients with large varices, and some specifically show no benefit of primary prophylaxis for patients with small or no varices $[4,6]$.

In order to prevent variceal bleeding in patients with cirrhosis, those patients at highest risk for bleeding must be identified. Clinical signs and symptoms such as ascites, thrombocytopenia, splenomegaly and Child-Pugh class do not adequately predict which patients will bleed [7-10]. Thus, the American College of Gastroenterology (ACG) and the American Association for the Study of Liver Disease (AASLD) have published guidelines recommending screening esophagogastroduodenoscopy (EGD) for GEV [11,12]. The initial guidelines, published in 1997 and 1998, recommended that once patients with large varices are identified, institution of primary prophylaxis is initiated. More recently, the 2007 guidelines have become more flexible in regard to initiating primary prophylaxis in patients with small varices with certain high-risk stigmata [13]. The recommendation to screen all cirrhotics without regard to clinical or laboratory findings remained, however.

While patients with large varices are started on lifelong primary prophylaxis, those with small or no varices must be monitored through surveillance endoscopy to assess the future risk of bleeding. The same guidelines that endorse universal screening for varices also recommend continued surveillance for varices; with repeat endoscopic surveillance recommended at 2-3 year intervals for the patients without GEV and 1-2 years for patients with small GEV.

Even though the guidelines for screening and the use of primary prophylaxis were originally published by the ACG and AASLD in 1997 and 1998, respectively, findings of regional studies, have shown that screening is underutilized $[14,15]$. There are no data regarding screening for high-risk varices and the institution of primary prophylaxis from across the U.S. or internationally. Thus, we explored practice patterns in a random sample of U.S. gastroenterologists and hepatologists. We examined whether differences exist in screening, surveillance and institution of primary prophylaxis between physicians in academic versus community practice, physicians who describe themselves as primarily gastroenterologists versus primarily hepatologists, and the physicians' number of years in practice.

\section{Materials and methods}

\subsection{Study design}

We conducted a national cross-sectional survey of academic and community gastroenterologists and hepatologists in the United States using a self-administered questionnaire (Appendix A). The survey was conducted between August 2003 and November 2003. Eligible participants had graduated from their training programme and 
were members of either the ACG or the AASLD. These two organizations were chosen in an effort to sample equal numbers of hepatologists and gastroenterologists. We used a random numbers generator to select 300 members of each of the above professional organizations.

\subsection{Survey distribution}

The survey (Appendix A), along with a cover letter explaining the goals of our project, was initially sent via facsimile to a random sample of 600 gastroenterologists and hepatologists in August 2003. We allowed a four-week response period for data collection and then refaxed the survey. Responses from partially completed questionnaires were not included in the analysis.

\subsection{Statistical analysis}

The collected data were analysed using the Statistical Analysis Software (SPSS 11.0, SPSS Inc., Chicago, IL). Descriptive data were presented as percentages and comparisons were performed by chi-square analysis or Fisher's exact test if applicable. Significance was defined at a $p$ value $\leq 0.05$.

\section{Results}

A total of 600 surveys were sent to randomly selected physicians and 191 questionnaires were returned (32\%). 180 (30\%) questionnaires were completed in their entirety whereas, 11 questionnaires were returned only partially completed and, as stated above, data derived from these were not included in the final analysis.

\subsection{Demographic data}

Respondent data is depicted in Table 1 . The mean age of the physicians was $48.9 \pm 10$ years and $87 \%$ of them were male. $50 \%$ physicians were based in the community (private practice) and $74 \%$ had been in practice for more than 10 years. $72 \%$ physicians describe themselves as gastroenterologists primarily, and $28 \%$ of them as hepatologists primarily. Overall, 37 states were represented with the greatest number of responses coming from New York (17), California (16), and Texas (12). Responses were also divided by region; there were 64 $(36 \%)$ responses from the Northeast, $65(36 \%)$ responses from the Southeast, $38(21 \%)$ responses from the Northwest, and $13(7 \%)$ responses from the Southwest.

As the responses to the survey were anonymous, we could not perform comparisons on all demographic variables between physicians that responded versus those that did not. However, we were able to ascertain if differences existed between the group as a whole and "responders" on certain variables. The gender and regional distribution of all survey recipients were recorded based on names and area codes. Overall, 91\% of the physicians who were faxed a survey were male versus $87 \%$ of responders $(p=\mathrm{NS})$. Regional distribution also did not differ significantly from the responders; $41 \%$ of the surveys were faxed to the Northeast region (36\% of responders), 31\% to the Southeast (36\% of responders), $19 \%$ to the Northwest (21\% of responders) and $9 \%$ to the Southwest (7\% of responders) ( $p=\mathrm{NS})$.

\subsection{Initial screening patterns-overall frequency of screening}

Physicians were asked to estimate how often they screen for varices in patients with cirrhosis without a history of prior bleeding. Only the physicians who screened consistently (defined as greater than $75 \%$ of the time) were counted as positive responses; $53 \%$ of them met these criteria $(n=95)$. Overall, by quartile, $53 \%$ physicians screened more than $75 \%$ of the time. $23 \%$ physicians screened between 50 and $75 \%$ of the time, $18 \%$ screened less than $50 \%$ of the time and $5 \%$ never screened. 
Comparisons were made between those who consistently screen versus those who do not screen consistently (Table 2). No differences were observed within practice setting and years in practice. Hepatologists screened more consistently than gastroenterologists (78\% vs. $45 \%$, $p<0.001)$.

\subsection{Initial screening patterns-types of patients screened}

The physicians who did report screening for varices at any point (i.e. frequency of screening anywhere from 1 to $100 \%, n=171$ ) were asked whether they screened all patients with cirrhosis or they screened only according to certain clinical characteristics. Overall, 106 of 171 physicians (62\%) reported that they screened all cirrhotics, whereas $38 \%$ physicians screened certain patients only on the basis of clinical signs and symptoms. There were no statistically significant differences among practice type, setting and years in practice. Those who screened only by clinical signs and symptoms reported the following as reasons to screen (more than one response was allowed): Child-Pugh class B/C 65\%, ascites 50\%, splenomegaly $47 \%$, telangectasia $12 \%$, thrombocytopenia $55 \%$, and other $7 \%$ (Fig. 1).

The comparison of physicians by practice type who screened selectively by clinical signs and symptoms showed significantly more hepatologists use thrombocytopenia as an indication for screening $\operatorname{EGD}(p=0.03)$.

When the responses to overall frequency of screening were compared with the responses to the types of patients screened, $76 \%(n=72)$ of the consistent screeners (screening in greater than $75 \%$ of cirrhotics) actually screened all cirrhotics regardless of the presence of certain clinical signs and symptoms. Thus, overall, only $40 \%$ of all physicians surveyed screen consistently and screen all patients with cirrhosis.

\subsection{Surveillance patterns: no varices}

The surveyed physicians were asked at what interval they would perform surveillance endoscopy in a patient with no varices. Overall, less than $1 \%$ physicians replied six-month intervals, $20 \%$ replied 1-year intervals, $41 \%$ replied 2-year intervals, $19 \%$ replied 3-year intervals, and $20 \%$ replied greater than 3 -year intervals.

\subsection{Surveillance patterns: small varices}

The surveyed physicians were asked at what interval they would perform surveillance endoscopy in a patient with small varices. Overall, 5\% physicians replied six-month intervals, $48 \%$ replied 1-year intervals, $25 \%$ replied 2-year intervals, $10 \%$ replied 3-year intervals, and $12 \%$ replied greater than 3-year intervals.

\subsection{Initiation of primary prophylaxis}

When the surveyed physicians were asked when they would start primary prophylaxis, the overall responses were: $6 \%$ would start primary prophylaxis in patients regardless of the presence or absence of varices, $40 \%$ in patients with small (less than $7 \mathrm{~mm}$ ) varices at endoscopy and 54\% in patients with large (greater than $7 \mathrm{~mm}$ ) varices at endoscopy.

The data comparing the timing of primary prophylaxis are shown in Table 3 . Significantly more hepatologists initiated primary prophylaxis for large varices only $(p<0.001)$. Significant differences were found by geographical analysis as well; more physicians from the Northwest initiated primary prophylaxis for large varices only when compared to other regions $(p=0.048)$. 


\subsection{Responders definition of primary prophylaxis and actual primary prophylaxis instituted}

When asked what the definition of primary prophylaxis was to them, in a format that allowed multiple answers, the surveyed physicians stated the following: $86.1 \%$ identified beta-blockers as primary prophylaxis; endoscopic variceal ligation (EVL), 28.3\%; betablockers and EVL, 25.6\%; nitrates, 11.7\%; sclerotherapy, 5.0\%; sclerotherapy and betablockers, 3.3\%; beta-blockers and nitrates, 20.0\%; one responder selected "other" and entered "portosystemic shunt", $0.6 \%$.

More academicians than community physicians identified endoscopic variceal ligation as a form of primary prophylaxis ( $36 \%$ vs. $21 \%, p=0.025)$. More physicians with less than or equal to 10 years' experience identified EVL ( $42 \%$ vs. $20 \%, p=0.017$ ) or EVL plus betablockers $(42 \%$ vs. $24 \%, p=0.03)$ as primary prophylaxis. There were also regional differences as more physicians in the Northwest and Southwest identified sclerotherapy ( $23 \%$ in SW vs. $2-5 \%$ in other regions, $p=0.014$ ) and variceal ligation ( $46 \%$ in $\mathrm{SW}, 42 \%$ in NW vs. $22-23 \%$ in other regions, $p=0.05$ ) as forms of primary prophylaxis.

Overall, the primary prophylaxis actually employed by the responding physicians were: beta-blockers, $84 \%$; EVL, 6\%; beta-blockers and EVL, 7\%; nitrates, $1 \%$, sclerotherapy $0.6 \%$, beta-blockers and sclerotherapy, $0.6 \%$; beta-blockers and nitrates, $0.6 \%$.

Significantly more academicians used beta-blockers as primary prophylaxis. More hepatologists used EVL. Physicians in practice less than or equal to 10 years were more likely to use a combination of EVL and beta-blockers $(17 \%$ vs. $3 \%, p=0.004)$ or nitrates $(4 \%$ vs. $0 \%, p=0.018)$ as primary prophylaxis.

\subsection{Limitations to adherence with published guidelines}

In the final question of the survey, physicians were asked whether there were any specific limitations or barriers to screening for GEV. The question allowed for multiple answers. $18.9 \%$ cited a lack of third party payer reimbursement; $8.9 \%$ were unfamiliar with the practice guidelines; $20 \%$ were uncertain about the benefit of primary prophylaxis; $20 \%$ cited the poor clinical features that predict GEV; $13.9 \%$ gave other responses.

Subgroup analysis showed several significant differences in barriers to screening. More community physicians were unfamiliar with the guidelines ( $14 \%$ vs. $3 \%, p=0.01)$. More gastroenterologists were unfamiliar with guidelines as well (12\% vs. $0 \%, p=0.01)$. Physicians with greater than 10 years' experience were more likely to question the benefits of screening ( $11 \%$ vs. $4 \%, p=0.05)$. Regional analysis showed that cost was a barrier in the Northeast and Southwest (28\% and 31\% respectively vs. $11-12 \%$ in other regions, $p=0.04$ ).

\section{Discussion}

According to project estimates, the number of patients with cirrhosis is expected to rise as a result of hepatitis $C$ and due to the effects of non-alcoholic fatty liver disease [16,17]. As a consequence of the increasing development of end-stage liver disease, the morbidity and mortality associated with complications from hepatocellular dysfunction and portal hypertension will be considerable. As "curative" treatment options for well-established cirrhosis are generally not available, therapeutic interventions usually focus on its complications after they have developed. Therefore, prevention is a cornerstone in the management of patients with cirrhosis. One such intervention, screening for high-risk varices, has been recommended by the ACG and the AASLD [11-13] Screening and surveillance for high-risk varices according to the published guidelines has been shown to be a cost-effective intervention [18]. Therefore, given the efficacy of primary prophylaxis [19] 
and the economical feasibility of screening, we would expect that the majority of practitioners embrace such a preventive strategy.

However, several regional studies had suggested that, despite these guidelines, screening for varices is under-implemented [20] Arguedas et al. [14] found that among patients presenting for liver transplant evaluation, only $46 \%$ had undergone any type of screening (endoscopic or radiological). Zaman et al. found that "screening for varices" was the major indication for EGD only $1.5 \%$ of the time according to the Clinical Outcomes Research Initiative [21] These studies, though, examined a limited number of patients who were prone to potential referral-bias owing to their regional nature. Their methodology did not permit an adequate understanding as to practice patterns of treating physicians and potential barriers to screening.

This study is the first to examine physician practice patterns across the United States in regard to screening for varices and implementation of primary prophylaxis. Members of two U.S.-based professional organizations (ACG and AASLD) were surveyed regarding their practice patterns concerning screening, surveillance and primary prophylaxis for GEV. Our response rate was considered adequate with 180 of 600 physicians (30\%) completing the entire questionnaire [22] In addition, there was consistency among the responses, which supports the fact that the results are representative of the current practice patterns of screening for varices and implementation of primary prophylaxis in the United States.

The most significant finding of our study is that only $53 \%$ of physicians consistently screen patients with cirrhosis. Of these, $76 \%$ screen all cirrhotics and $24 \%$ screen only if there are clinical or laboratory indicators suggesting portal hypertension; $5 \%$ of physicians never recommend screening at all. Therefore, only $40 \%$ of physicians adhere to published recommendations leaving a large proportion of patients with high-risk varices that are not identified, and therefore, remain unprotected.

When comparisons were made between physicians who consistently screen and those who do not, there was a statistically significant higher proportion of hepatologists who recommended screening compared to gastroenterologists (78\% vs. 45\%, respectively). We speculate this may be due, in part, to greater familiarity with published guidelines as no hepatologists responded to a "lack of awareness" as a barrier to screening, whereas $12 \%$ of gastroenterologists did. In addition, academic physicians were more familiar with practice guidelines. This could be related to the fact that most hepatologists practice in the academic setting. "Lack of reimbursement" was cited by $20 \%$ of physicians as a potential barrier for screening. According to data from our institution, pre-certification prior to EGD is usually not necessary in patients with cirrhosis, thus, the procedure is usually reimbursed.

In patients in whom an initial endoscopy did not reveal varices, surveillance patterns did not differ significantly according to practice type and setting, years in practice and geographical location. A "trend" towards more frequent screening among hepatologists and practitioners in the Southwestern United States was observed. We do not know the significance of this finding and it could reflect alpha statistical error. In patients in whom an initial screening endoscopy revealed small varices, surveillance patterns did not differ significantly according to practice type and setting or years in practice. Physicians in the Northeastern United States tended to perform surveillance at greater intervals than physicians in the rest of the country.

Non-selective beta-blockers were recommended as the initial primary prophylaxis modality by $86 \%$ of respondents with the majority of the remaining physicians recommending endoscopic variceal ligation. Interestingly, we found that primary prophylaxis is instituted in patients with small varices by the majority of gastroenterologists (49\%) whereas the majority of hepatologists $(80 \%)$ implemented primary prophylaxis in patients with large 
varices. A recent placebo, controlled study by Merkel et al. [5] found that the use of nadolol in patients with small varices was associated with a lower "variceal growth rate" (9/83 patients developed large varices) compared to 29 of 78 patients on placebo developing highrisk varices. In addition, this study found that the risk of a first variceal bleed was significantly lower in patients on nadolol. These findings contrast with those found by several authors over the past two decades which have shown the benefit of primary prophylaxis in patients with large varices [4]. In addition, a recent study demonstrated that non-selective beta-blockers do not prevent the development of varices in patients with portal hypertension [6].

These data also are taken into account in the 2007 updated AASLD guidelines. All patients with cirrhosis should be screened for GEV regardless of clinical signs or symptoms. Primary prophylaxis is still not recommended to prevent the formation of varices. Non-selective betablockers should be used for high-risk small varices that have not bled. Non-selective betablockers may be used for low risk small varices that have not bled, although the long-term benefit of this strategy has not been established. EVL should be reserved for large varices that have a high risk of haemorrhage or large varices in those patients who cannot tolerate a beta-blocker. Nitrates (alone or with beta-blockers), shunt therapy, or sclerotherapy should not be used as primary prophylaxis [13].

Our study has several potential limitations. While the study was conducted five years ago, no studies to reflect current practice have been done since that time, thus these data represent the most up to date information available in the United States or internationally. The survey was done with the 1997-1998 guidelines in mind; however, the new guidelines do not change the overall results or interpretation of the survey, especially in regard to the lack of screening given to all cirrhotics and the warning against the use of markers for portal hypertension that are neither sensitive nor specific for varices.

Although this survey data is from American physicians, the guidelines were published in journals with a widespread international audience. The AASLD and the ACG and their respective publications, Hepatology and the American Journal of Gastroenterology, reflect international opinions from international panels of experts. The guidelines were meant to apply to all healthcare professionals caring for patients with liver disease, regardless of country of origin and thus the survey results should be of interest to all practitioners.

The label of hepatologist vs. gastroenterologist may contribute to some bias in the survey responses. A "Hepatologist" was defined as a physician whose patient population consists mainly of patients with liver disease (Appendix A, question 5). Respondents self-determined their status. It is possible that those with a greater interest in liver disease were more likely to define themselves as hepatologists and adhere to the guidelines more closely. Nevertheless, as the overall number of gastroenterologists significantly outnumbers the hepatologists in the United States and the majority of patients with cirrhosis are cared for by community gastroenterologists, the practice patterns of all respondents remain important.

Recently, there has been a Certificate of Added Qualification for Transplant Hepatology sanctioned by the American Board of Internal Medicine and the advent of transplant hepatology fellowship programmes. Prior to the 1990's, formal training in hepatology was not widespread and expertise was based on clinical and/or research experience. While scattered hepatology fellowship programmes did exist, their numbers paled in comparison to gastroenterology training programs [23] Given the average age of the respondents, 49, most would have finished their fellowships at least 15 years ago, before such dedicated training was widespread. 
Other limitations within our study are inherent to survey studies in general. First, while the response rate was only $30 \%$, this is similar or better than response rates achieved in other published surveys [24-26] More critical than response rate, however, is the degree to which non-respondents are similar to respondents. Based on the available data, certain demographic features were similar between all survey recipients and those who chose to respond. If these groups are dissimilar, a survey study can be biased. Thus, the second possible limitation of our survey study is that only highly motivated practitioners answered the questionnaire thereby introducing this bias. In a worst case scenario, physicians may answer what they "consider correct" as opposed to what they really implement in clinical practice. Still, these concerns do not explain the relatively low proportion of physicians who consistently screen for varices. We would expect that introduction of bias would have "falsely" increased the proportion of physicians who screen. This only serves to underscore the relative lack of compliance with the established guidelines.

In conclusion, endoscopic screening for varices in patients with cirrhosis in the United States is under-implemented by both hepatologists and gastroenterologists. Failure to screen leaves a considerable proportion of patients at risk for variceal bleeding and contributes to significant morbidity, mortality and healthcare costs. The solution to this problem lies in continuing medical education for physicians that stresses not only what the guidelines are but also why primary prophylaxis is vitally important to our patients. Another barrier, third party payer reimbursement, varies by region and is certainly affected by individual managed care organizations. This barrier, however, may become an incentive as more managed care organizations investigate merit pay options and base their reimbursement on compliance with the established guidelines. Until further information is available regarding whether pharmacological agents prevent the development and/or growth of varices or until reliable clinical signs and symptoms accurately predict which patients will bleed, endoscopic screening for varices remains an acceptable, cost-effective intervention.

\section{References}

1. Smith JL, Graham DY. Variceal hemorrhage: a critical evaluation of survival analysis. Gastroenterology. 1982; 82:968-73. [PubMed: 7037525]

2. de Franchis R. Evolving consensus in portal hypertension. Report of the Baveno IV consensus workshop on methodology of diagnosis and therapy in portal hypertension. J Hepatol. 2005; 43:167-76. [PubMed: 15925423]

3. Lowe RC, Grace ND. Primary prophylaxis of variceal hemorrhage. Clin Liver Dis. 2001; 5:665-76. [PubMed: 11565136]

4. D’Amico G, Pagliaro L, Bosch J. Pharmacological treatment of portal hypertension: an evidencebased approach. Semin Liver Dis. 1999; 19:475-505. [PubMed: 10643630]

5. Merkel C, Marin R, Angeli P, Zanella P, Felder M, Bernardinello E, et al. A placebo-controlled clinical trial of nadolol in the prophylaxis of growth of small esophageal varices in cirrhosis. Gastroenterology. 2004; 127:476-84. [PubMed: 15300580]

6. Groszmann RJ, Garcia-Tsao G, Bosch J, Grace ND, Burroughs AK, Planas R, et al. Beta-blockers to prevent gastroesophageal varices in patients with cirrhosis. N Engl J Med. 2005; 353:2254-61. [PubMed: 16306522]

7. de Franchis R, Primignani M. Natural history of portal hypertension in patients with cirrhosis. Clin Liver Dis. 2001; 5:645-63. [PubMed: 11565135]

8. Ng FH, Wong SY, Loo CK, Lam KM, Lai CW, Cheng CS. Prediction of oesophagogastric varices in patients with liver cirrhosis. J Gastroenterol Hepatol. 1999; 14:785-90. [PubMed: 10482429]

9. Chalasani N, Inperiale TF, Ismail A, Sood G, Carey M, Wilcox CM, et al. Predictors of large esophageal varices in patients with cirrhosis. Am J Gastroenterol. 1999; 94:3285-91. [PubMed: 10566731]

Dig Liver Dis. Author manuscript; available in PMC 2012 March 2. 
10. Qamar AA, Grace ND, Groszmann RJ, Garcia-Tsao G, Bosch J, Burroughs AK, et al. Platelet count is not a predictor of the presence or development of gastroesophageal varices in cirrhosis. Hepatology. 2008; 47:153-9. [PubMed: 18161700]

11. Grace ND. Diagnosis and treatment of gastrointestinal bleeding secondary to portal hypertension. American College of Gastroenterology Practice Parameters Committee. Am J Gastroenterol. 1997; 92:1081-91. [PubMed: 9219775]

12. Grace ND, Groszmann RJ, Garcia-Tsao G, Burroughs AK, Pagliaro L, Makuch, et al. Portal hypertension and variceal bleeding: an AASLD single topic symposium. Hepatology. 1998; 28:868-80. [PubMed: 9731585]

13. Garcia-Tsao G, Sanyal AJ, Grace ND, Carey W. Prevention and management of gastroesophageal varices and variceal hemorrhage in cirrhosis. Hepatology. 2007; 46:922-38. [PubMed: 17879356]

14. Arguedas MR, McGuire BM, Fallon MB, Abrams GA. The use of screening and preventive therapies for gastroesophageal varices in patients referred for evaluation of orthotopic liver transplantation. Am J Gastroenterol. 2001; 96:833-7. [PubMed: 11280560]

15. Hapke RJ, Zaman A, Benner KG, Rosen HR, Flora K. Prevention of first variceal hemorrhage-a survey of community practice patterns. Hepatology. 1997; 26:A30. [Abstract].

16. Choudhury J, Sanyal AJ. Clinical aspects of fatty liver disease. Semin Liver Dis. 2004; 24:349-62. [PubMed: 15605303]

17. Davis GL, Albright JE, Cook SF, Rosenberg DM. Projecting future complications of chronic hepatitis $C$ in the United States. Liver Transpl. 2003; 9:331-8. [PubMed: 12682882]

18. Arguedas MR, Heudebert GR, Eloubeidi MA, Abrams GA, Fallon MB. Cost-effectiveness of screening, surveillance, and primary prophylaxis strategies for esophageal varices. Am J Gastroenterol. 2002; 97:2441-52. [PubMed: 12358270]

19. Shahi HM, Sarin SK. Prevention of first variceal bleed: an appraisal of current therapies. Am J Gastroenterol. 1998; 93:2348-58. [PubMed: 9860391]

20. Zaman A, Hapke RJ, Flora K, Rosen HR, Benner KG. Changing compliance to the American College of Gastroenterology guidelines for the management of variceal hemorrhage: a regional survey. Am J Gastroenterol. 2004; 99:645-9. [PubMed: 15089896]

21. Zaman A, Flora K, Benner KG. Endoscopic screening for varices in cirrhotics: preliminary data from a national endoscopic database (the CORI project). Hepatology. 1998; 28:A573. [Abstract].

22. McMahon SR, Iwamoto M, Massoudi MS, Yusuf HR, Stevenson JM, David F, et al. Comparison of e-mail, fax, and postal surveys of pediatricians. Pediatrics. 2003; 111:e299-303. [PubMed: 12671142]

23. Bacon BR. Hepatology: where do we go from here? Gastroenterology. 2006; 131:1373-4. [PubMed: 17064705]

24. Fendrick AM, Hirth RA, Chernew ME. Differences between generalist and specialist physicians regarding Helicobacter pylori and peptic ulcer disease. Am J Gastroenterol. 1996; 91:1544-8. [PubMed: 8759658]

25. Falk GW, Ours TM, Richter JE. Practice patterns for surveillance of Barrett's esophagus in the United States. Gastrointest Endosc. 2000; 52:197-203. [PubMed: 10922091]

26. Charpentier KP, Mavanur A. Removing patients from the liver transplant wait list: a survey of US liver transplant programs. Liver Transpl. 2008; 14:303-7. [PubMed: 18306339]

\section{Appendix A}

\section{What is your age?}

2. What is your gender?

3. In which State do you currently practice?

4. Setting of current practice:

i. Community-based

ii. Academic-based

Dig Liver Dis. Author manuscript; available in PMC 2012 March 2. 
5. Type of current practice ( $>50 \%$ of patients seen):

i. Primarily Gastroenterology

ii. Primarily Hepatology

6. Years in practice (since completion of fellowship):

i. Less than or equal to 10 years

ii. Greater than 10 years

7. Do you screen for varices in patients without prior bleeding?

i. Consistently (greater than $75 \%$ of the time)

ii. Frequently (between $50-75 \%$ of the time

iii. Infrequently (between $1-49 \%$ of the time)

iv. Never (Skip questions 8,9 and 10)

8. Who do you screen for varices?

i. All patients with cirrhosis

ii. Patients with cirrhosis and (circle all that apply)

- Child-Pugh class B/C

- ascites

- splenomegaly

- telangiectasias

- thrombocytopenia

- other; please specify:

9. In patients with no varices on initial screening, at what interval would you perform surveillance endoscopies?

i. 6-month intervals

ii. 1-year intervals

iii. 2-year intervals

iv. 3-year intervals

v. Greater than 3 years; please specify:

10. In patients with small varices (grade I, less than $7 \mathrm{~mm}$ ), at what interval would you perform subsequent surveillance endoscopies?

i. 6-month intervals

ii. 1-year intervals

iii. 2-year intervals

iv. 3-year intervals

v. Greater than 3 years; please specify:

11. When would you institute primary prophylaxis?

i. Regardless of the absence or presence of varices

Dig Liver Dis. Author manuscript; available in PMC 2012 March 2. 

ii. Small varices at endoscopy
iii. Large varices at endoscopy (greater than $7 \mathrm{~mm}$ )

12. What is your definition of primary prophylaxis (circle all that apply) ?

i. Beta-blockers

ii. Endoscopic variceal ligation

iii. Endoscopic variceal ligation plus beta-blockers

iv. Nitrates

v. Sclerotherapy

vi. Sclerotherapy plus beta-blockers

vii. Beta-blockers and nitrates

viii. Other

13. What type of primary prophylaxis do you recommend initially?

i. Beta-blockers

ii. Endoscopic variceal ligation

iii. Endoscopic variceal ligation plus beta-blockers

iv. Nitrates

v. Sclerotherapy

vi. Sclerotherapy plus beta-blockers

vii. Beta-blockers and nitrates

14. What are the major reasons you do not screen for varices (circle all that apply)?

i. Lack of third-party payer reimbursement

ii. Unfamiliarity about practice guidelines that recommend screening

iii. Uncertainty about benefit of primary prophylaxis

iv. Poor clinical features that predict the presence of varices

v. Other; please specify: 


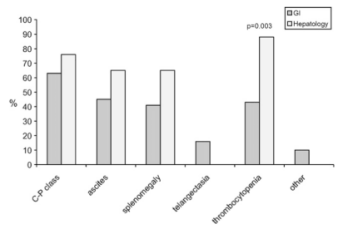

Fig. 1.

Selective screening: GI vs. Hepatology. 
Table 1

Survey demographics.

\begin{tabular}{lcc}
\hline & $N$ & $\%$ \\
\hline Completed surveys & 180 & $30^{a}$ \\
Age (years) & $48.9 \pm 10$ & \\
Sex (Male) & 156 & 87 \\
Practice Setting (Community) & 90 & 50 \\
Practice Type (Primarily Gastroenterologists) & 130 & 72 \\
Practice Length (>10 years) & 133 & 74 \\
Region & & \\
Northeast & 64 & 36 \\
Southeast & 65 & 36 \\
Northwest & 38 & 21 \\
Southwest & 13 & 7 \\
\hline
\end{tabular}

${ }^{a} 600$ total surveys distributed. 
Table 2

Screening frequency.

\begin{tabular}{llll}
\hline & Screen consistently $(\mathbf{2 7 5} \text { of the time })^{\boldsymbol{a}}$ & Do not screen consistently/never screen $\boldsymbol{a}^{\boldsymbol{p} \text { value }}$ \\
\hline Community & 52 & 48 & NS \\
Academic & 56 & 44 & \\
Gastroenterology & 45 & 55 & $<0.001$ \\
Hepatology & 78 & 22 & \\
$\leq 10$ years in practice & 63 & 37 & NS \\
$>10$ years in practice & 51 & 49 & \\
NE & 53 & 47 & NS \\
SE & 59 & 41 & \\
NW & 40 & 60 & \\
SW & 77 & 23 & \\
\hline
\end{tabular}

${ }^{a}$ Data are presented as percentages. 
Table 3

Initiation of primary prophylaxis.

\begin{tabular}{lllll}
\hline & Regardless & Small varices & Large varices & $\boldsymbol{p}$ value \\
\hline Community & 7 & 47 & 46 & NS \\
Academic & 4 & 33 & 63 & \\
Gastroenterology & 7 & 49 & 44 & $<0.001$ \\
Hepatology & 2 & 18 & 80 & \\
$\leq 10$ years in practice & 7 & 43 & 50 & NS \\
$>10$ years in practice & 4 & 33 & 63 & \\
NE & 8 & 33 & 59 & 0.048 \\
SE & 6 & 55 & 39 & \\
NW & 3 & 26 & 71 & \\
SW & 8 & 38 & 54 & \\
\hline
\end{tabular}

Data are presented as percentages. 